Check for updates

Cite this: Phys. Chem. Chem. Phys. 2021, 23, 26451

Received 1st September 2021, Accepted 3rd November 2021 DOI: $10.1039 / \mathrm{d} 1 \mathrm{cp} 04005 \mathrm{~g}$

rsc.li/pccp

\section{Mechanistic insights into the effects of key mutations on SARS-CoV-2 RBD-ACE2 binding}

\author{
Abhishek Aggarwal, (D) $\dagger^{a}$ Supriyo Naskar, iD $\dagger^{a}$ Nikhil Maroli, $\dagger^{a}$ Biswajit Gorai, \\ Narendra M. Dixit ${ }^{\mathrm{bc}}$ and Prabal K. Maiti (D) *a
}

Some recent SARS-CoV-2 variants appear to have increased transmissibility compared to the original strain. An underlying mechanism could be the improved ability of the variants to bind receptors on the target cells and infect them. In this study, we provide atomic-level insights into the binding of the receptor binding domain (RBD) of the wild-type SARS-CoV-2 spike protein and its single (N501Y), double (E484Q, L452R) and triple (N501Y, E484Q, L452R) mutated variants to the human ACE2 receptor. Using extensive all-atom molecular dynamics simulations and advanced free energy calculations, we estimate the associated binding affinities and binding hotspots. We observe significant secondary structural changes in the RBD of the mutants, which lead to different binding affinities. We find higher binding affinities for the double (E484Q, L452R) and triple (N501Y, E484Q, L452R) mutated variants than for the wild type and the N501Y variant, which could contribute to the higher transmissibility of recent variants containing these mutations.

\section{Introduction}

The COVID-19 pandemic has caused more than 4.5 million deaths so far. The severe acute respiratory syndrome coronavirus 2 (SARS-CoV-2), the causative agent of COVID-19, attaches to the target host cells via its spike protein, called $\mathrm{S}$, by binding to the angiotensin-converting enzyme 2 (ACE2) receptor, mainly expressed in the lungs, ${ }^{1-4}$ and causes the infection. The binding of $\mathrm{S}$ to ACE2 triggers conformational changes in $\mathrm{S}$ from its metastable prefusion state to a stable post-fusion state. ${ }^{5}$ The spike protein is divided into two subunits, S1 and $\mathrm{S} 2$, in the prefusion stage. The receptor binding domain (RBD) in $\mathrm{S} 1$ is responsible for the binding with ACE2, whereas S2 mediates the subsequent fusion of viral and cell membranes, allowing viral entry. ${ }^{5}$ Characterizing the molecular details of the interaction between the RBD and ACE2 plays an important role in understanding the process of SARS-CoV-2 infection in target cells. This understanding may also help in explaining the improved transmissibility that is observed in SARS-CoV-2 variants that carry mutations potentially affecting RBD-ACE2 binding.

SARS-CoV-2 belongs to the betacoronavirus genus, which includes SARS-CoV and the middle-east respiratory syndrome

\footnotetext{
${ }^{a}$ Center for Condensed Matter Theory, Department of Physics, Indian Institute of Science, Bangalore 560012, India. E-mail: maiti@iisc.ac.in; Tel: 091-80-2293-2865

${ }^{b}$ Department of Chemical Engineering and Centre for Biosystems Science and Engineering, Indian Institute of Science, Bangalore 560012, India

${ }^{c}$ Centre for Biosystems Science and Engineering, Indian Institute of Science, Bangalore 560012, India

$\dagger$ Equal contribution.
}

virus (MERS). ${ }^{6}$ SARS-CoV-2 and SARS-CoV have a high sequence similarity of $\sim 80 \%$. SARS-CoV-2, however, has spread far more than SARS-CoV, which may be due to the higher binding energy of SARS-CoV-2 with ACE2. ${ }^{7,8}$ More recently, several variants of SARS-CoV-2 carrying mutations in the RBD, and other regions of $\mathrm{S}$, have been reported to have heightened transmissibility and can cause infections that differ in severity among individuals. ${ }^{9,10}$ The double mutated variant B.1.617 has already caused a second wave of COVID cases in India. ${ }^{11}$ There have been several reports from the Indian sub-continent about the potential infection and death rate or severe complications caused by these mutated variants. ${ }^{12}$ The underlying molecular mechanism behind the binding of these variants with human ACE2 receptor is still unknown.

In this study, we estimate the binding energy between ACE2 and the RBD of the SARS-CoV-2 wild-type, and that of its single (N501Y), double (E484Q and L452R) and triple (E484Q, L452R, and N501Y) mutated variants. We have considered the two mutations E484Q and L452R seen in the variant B.1.617 responsible for the second wave in India. ${ }^{13}$ We considered these mutations along with the B.1.1.7 N501Y variant, one of the early mutations argued to have caused increased transmissibility. ${ }^{14}$ We calculate the binding energy differences due to these mutations and elucidate the underlying molecular mechanisms using all-atom molecular dynamics simulations. We examine the structural rearrangements of the RBD in the mutated variants leading to these differences. We also employ the ASGB method for a quantitative analysis of the binding mechanisms. 


\section{Materials and methods}

\subsection{Simulation set up}

The three-dimensional structures of ACE2 and RBD domains of SARS-CoV-2 were obtained from the protein data bank PDB ID: 6M17. ${ }^{15}$ We studied various mutated variants of this structure as listed in Table 1 . The mutated structures were prepared using CHIMERA software package.

Using the xLEaP module of AmberTools $18,{ }^{16}$ the protein structures were enclosed in a water box with a buffer of $1.5 \mathrm{~nm}$ in all three directions. The systems were then charge neutralized by adding an appropriate number of $\mathrm{Na}^{+}$ions. Additional $\mathrm{Na}^{+}$ions and an equal number of $\mathrm{Cl}^{-}$ions were further added to achieve a physiological salt concentration of $150 \mathrm{mM}$. The TIP3P water model $^{17}$ was used for solvating the proteins. The ff99sb-ildn ${ }^{18}$ force field was used to represent the inter- and intra-molecular interactions of the protein atoms. Interactions involving ions were described using the Joung Cheatham parameter set. ${ }^{19}$

\subsection{MD simulation protocol}

The solvated systems were initially energy minimized for 5000 steps using the steepest descent algorithm. The minimized structures were then heated from 0 to $300 \mathrm{~K}$ within a period of $50 \mathrm{ps}$ with the solute atoms held fixed by a harmonic potential of strength $20 \mathrm{kcal} \mathrm{mol}^{-1} \AA^{2}$. After heating, the systems were subjected to a 2 ns long unrestrained equilibration. Finally, the equilibrated structures were subjected to $200 \mathrm{~ns}$ long production runs in the $N V T$ ensemble. All the simulations were performed using the AMBER18 simulation package. ${ }^{16}$ Periodic boundary conditions were employed in all the three directions. SHAKE constraints were used for all bonds containing hydrogen allowing the use of a time step of $2 \mathrm{fs}$. A Langevin thermostat with a collision frequency of $2 \mathrm{ps}^{-1}$ was used for temperature regulation while an isotropic Berendsen barostat with a coupling constant of 0.5 ps was used to regulate pressure in the NPT simulations. A cut-off of $10 \AA$ was used to compute the short-range Lennard-Jones (LJ) interaction, while PME (Particle Mesh Ewald ${ }^{20}$ ) was employed for the calculation of the long-range electrostatic interaction. The snapshots were visualized using $\mathrm{VMD}^{21}$ for analysis.

\subsection{Binding energy calculation}

The MMPBSA method ${ }^{22,23}$ employed in the MMPBSA.py module of AMBER18 was used to calculate the binding energies of the different ACE2-RBD complexes. The binding free energy difference $\left(\Delta G_{\text {bind }}\right)$ of the SARS-CoV-2 RBD and ACE2 complex formation is calculated as follows: ${ }^{24}$

$$
\Delta G_{\text {bind }}=G_{\text {complex }}-G_{\mathrm{RBD}}-G_{\mathrm{ACE} 2}
$$

Table 1 List of systems studied in this work

\begin{tabular}{ll}
\hline Mutation variant & Mutations \\
\hline Wild type & - \\
Single mutation & $\mathrm{N} 501 \mathrm{Y}$ \\
Double mutation & $\mathrm{E} 484 \mathrm{Q}, \mathrm{L452R}$ \\
Triple mutation & $\mathrm{N} 501 \mathrm{Y}, \mathrm{E} 484 \mathrm{Q}, \mathrm{L} 452 \mathrm{R}$
\end{tabular}

where $G_{\text {complex }}, G_{\mathrm{RBD}}$, and $G_{\mathrm{ACE} 2}$ represent the free energies of the RBD-ACE2 complex, individual RBD and individual ACE2 receptor, respectively. Eqn (1) can be decomposed into different interactions and can be written as

$$
\Delta G_{\mathrm{bind}}=\Delta H-T \Delta S \approx \Delta E_{\mathrm{MM}}+\Delta G_{\mathrm{sol}}-T \Delta S
$$

Here, $\Delta E_{\mathrm{MM}}$ is the change in gas-phase molecular mechanics energy, $\Delta S$ is the entropy change (which has been neglected in this work), and $\Delta G_{\text {sol }}$ represents the solvation free energy change upon ligand binding. $\Delta E_{\mathrm{MM}}$ can be further written as

$$
\Delta E_{\mathrm{MM}}=\Delta E_{\text {bonded }}+\Delta E_{\text {elec }}+\Delta E_{\mathrm{vdW}}
$$

Here $\Delta E_{\text {bonded }}, \Delta E_{\text {elec }}$, and $\Delta E_{\mathrm{vdw}}$ represent the change in bonded energies (bond, angle, and dihedral), electrostatic energies, and van der Waals energies upon ligand binding, respectively. $\Delta G_{\text {sol }}$ is the sum of the nonpolar solvation energy $\left(\Delta G_{\mathrm{SASA}}\right)$ and electrostatic solvation energy $\left(\Delta G_{\mathrm{PB}}\right)$, computed using the Poisson-Boltzmann method. $\Delta G_{\mathrm{SASA}}$ can be written as

$$
\Delta G_{\mathrm{SASA}}=\gamma \mathrm{SASA}+\beta
$$

Here, $\gamma\left(=0.00542 \mathrm{kcal} \AA^{2}\right)$ is the surface tension, while $\beta=$ $0.92 \mathrm{kcal} \mathrm{mol}^{-1}$, and SASA represents the solvent-accessible surface area of the molecule.

\subsection{Hotspot prediction by the ASGB method}

Alanine scanning using the MM/GBSA (ASGB) method is used during the last $10 \mathrm{~ns}$ of the $200 \mathrm{~ns}$ long MD simulation of each of the four RBD-ACE2 complex structures. In this method, all the residues lying within the $5 \AA$ range of the protein-protein interaction interface are mutated. We use the ASGB method with dielectric constants of 1,3 , and 5 for nonpolar, polar, and charged residues, ${ }^{24-26}$ respectively. The binding free energy difference due to point mutation was calculated by using the following relations:

$$
\begin{gathered}
\Delta \Delta G_{\text {blind }}^{x \rightarrow a}(\mathrm{ASGB}) \approx \Delta G_{\mathrm{sol}}^{x \rightarrow a}+\Delta G^{x \rightarrow a} \\
\Rightarrow \Delta \Delta G_{\mathrm{blind}}^{x \rightarrow a}(\mathrm{ASGB}) \approx \Delta G_{\mathrm{sol}}^{x \rightarrow a}+\left(\Delta E_{\text {elec }}^{x-\mathrm{ACE} 2}+\Delta E_{\mathrm{vdW}}^{x-\mathrm{ACE} 2}\right)
\end{gathered}
$$

Here, $\Delta \Delta G_{\text {blind }}^{x \rightarrow a}(\mathrm{ASGB})$ is the change in the binding free energy difference upon ligand binding due to a single pointmutation in RBD, while $\Delta G_{\text {sol }}^{x \rightarrow a}$ is the change in the solvation free energy difference upon ligand binding due to single point-mutation in RBD. $\Delta E_{\text {elec }}^{x-A C E 2}$ and $\Delta E_{\mathrm{vdW}}^{x-\mathrm{ACE}^{2}}$ represent the difference in electrostatic and van der Waals energies between the mutated residue $(a)$ in RBD with ACE2 to that of the premutated residue $(x)$ in RBD with ACE2.

\section{Results and discussion}

The structural stability of wild type, and the single (N501Y), double (E484Q and L452R), and triple (N501Y, E484Q, and L452R) mutants has been evaluated by analysing the RMSD 
fluctuations of backbone atoms and $\mathrm{C} \alpha$ atoms (Fig. 1). The single (N501Y) and double (E484Q, L452R) mutated variants have a similar RMSD to wild type, while they show lower fluctuations than the triple (N501Y, E484Q, and L452R) mutated variant. The RMSF fluctuations of all the 4 studied variants of SARS-CoV-2 RBD are shown in Fig. 2(a). Overall, the mutated RBD shows higher fluctuations compared to wild type RBD. The loop region of the RBD of all the SARS-CoV-2 variants have higher fluctuations compared to the other parts of the structures. Snapshots provided in Fig. 2(b-f) show that the loops formed by the residues 362-371, 380-390, 410-420, 425-432, and 474-484 exhibit higher RMSF compared to the rest of the RBD structure.

We have calculated the average structures of the wild-type and mutated variants of the SARS-CoV-2 systems using the last 100 ns MD simulation. In Fig. 3, we present the average superimposed three-dimensional structures of the single (N501Y), double (E484Q and L452R), and triple (N501Y, E484Q, and L452R) mutated variants with wild type SARS-CoV-2 RBD. The superposed 3-D structures indicate higher movements of the chains, including the backbone atoms of the various mutated variants than those of the wild type RBD. The N501Y point mutation lies near the binding site to ACE2, while the other point mutation sites (E484Q and L452R) lie away from the binding sites. Fig. 4 shows the secondary structural changes of RBD for all the variants. The wild type RBD shows a transition from $25.1 \%$ sheet, $25.7 \%$ turn, and $49.2 \%$ coil to $3.3 \%$ helix, $25.8 \%$ sheet, $22.5 \%$ turn, and $48.4 \%$ coil during 200 ns simulation. The introduction of N501Y mutation has changed the final secondary structure content to $2.7 \%$ helix, $30.6 \%$ sheet, $24.0 \%$ turn, $40.4 \%$ coil, and 2.2\% 3-10 helix. In comparison, the double (E484Q and L452R) and triple (N501Y, E484Q, and L452R) mutants show $24.7 \%$ sheet, $22.5 \%$ turn, $50.5 \%$ coil, and $2.2 \%$ 3-10 helix, and 3.3\% helix, $24.2 \%$ sheet, $23.6 \%$ turn, and $48.9 \%$ coil after $200 \mathrm{~ns}$, respectively. There are more transitions in the secondary structure for the triple (N501Y, E484Q, and L452R) mutated variant than the double mutant (E484Q, L452R) and the wild-type. This indicates the structural rearrangements induced by these mutations even while not in direct contact with ACE2. In Fig. 5(a), we show the superimposed average structures of wild-type (red) and double mutated (blue) SARS-CoV-2 RBD-ACE2 complex. A few residues of the $\mathrm{N}$-terminal of ACE2 and another few residues of RBD in their vicinity are shown in solid representation, while other residues are shown in transparent representation. As can be seen from the enlarged structures, the double (E484Q and L452R) mutated SARS-CoV-2 RBD orients towards the $\alpha$-helix region of the N-terminal of ACE2. Similarly, the triple (N501Y, E484Q, and L452R) mutated RBD also orients towards the $\alpha$-helix region of the N-terminal of ACE2 (Fig. 5(b)). To obtain a quantitative support for this observation, we computed the average distance between the highlighted residues (first 15 residues of N-terminal of ACE2 and residues 469 to 489 for all the variant SARS-CoV-2 RBD studied in this work). The average distance is found to be $12.59 \AA$ for wild type, while the distances are $12.72 \AA, 10.85 \AA$, and $11.06 \AA$ for single, double, and triple mutants respectively (Fig. 5(c)). Thus, our analysis shows that the double (E484Q and L452R) and triple
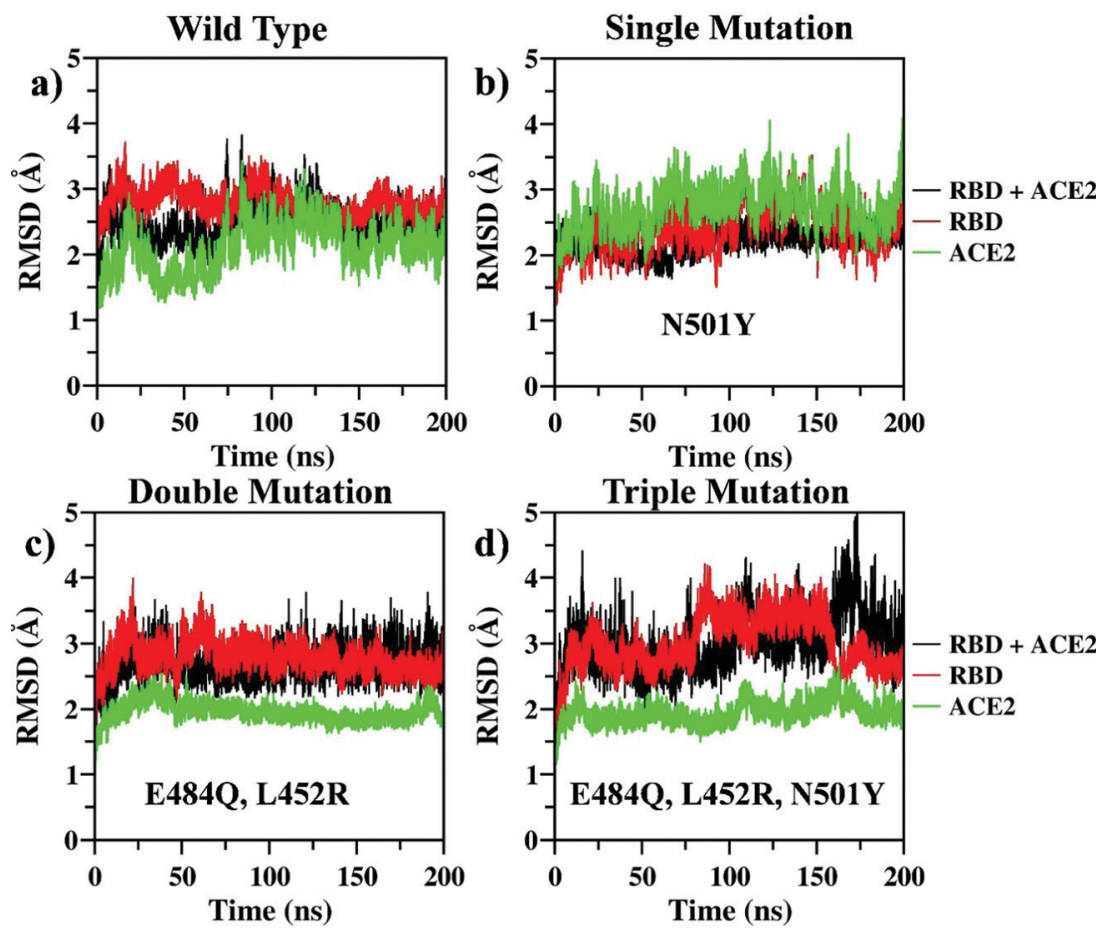

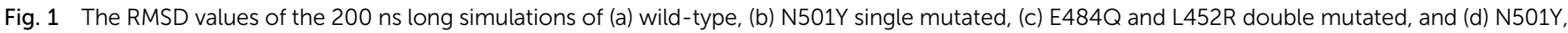

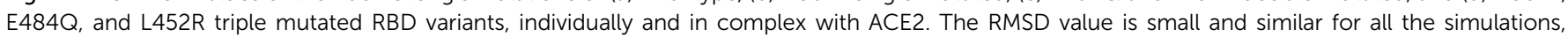
indicating the stability of the simulations performed. 
(a)

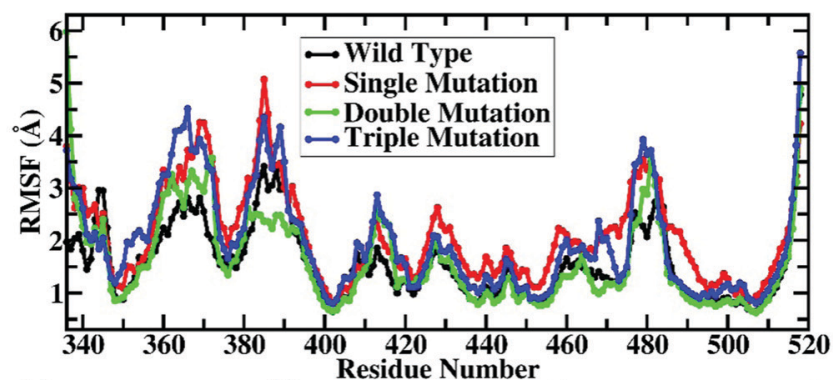

(b)

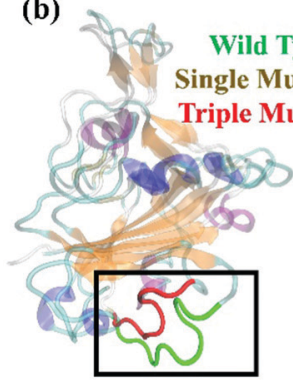

Residue Range 362-371 (c)

(c)

e

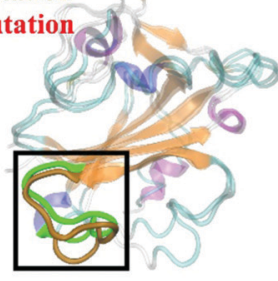

Residue Range 380-390 (d)

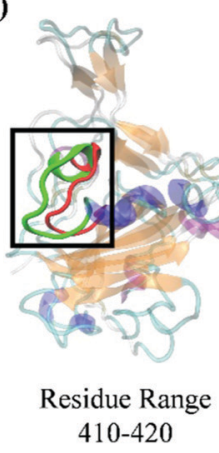

(e)

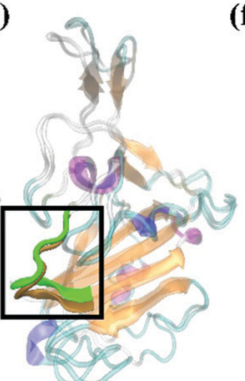

Residue Range 425-432 (f)
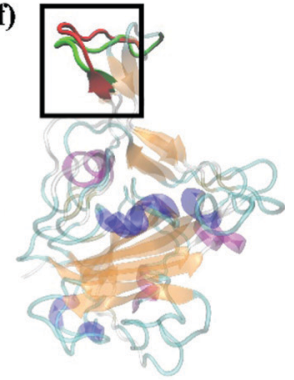

Residue Range 474-484

Fig. 2 (a) A comparison of residue-wise RMSF values for wild-type, N501Y single mutated, E484Q and L452R double mutated, and N501Y, E484Q, and L452R triple mutated variant RBD. (b-f) Superimposed 3D-structures of different residue regions of N501Y single mutated (highlighted in brown color) RBD or N501Y, E484Q, and L452R triple mutated (highlighted in red color) variant with wild-type (highlighted in green color) RBD having high RMSF values.

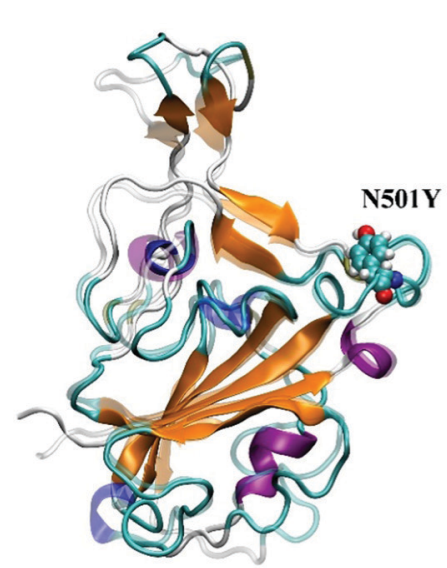

(a) Single Mutation

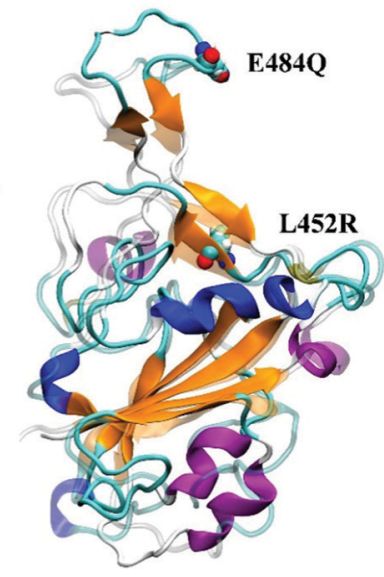

(b) Double Mutation

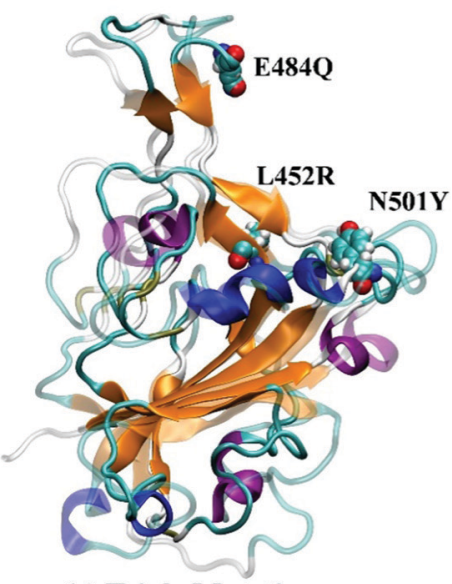

(c) Triple Mutation

Fig. 3 Superimposed structures (averaged during the last 100 ns of MD simulations) of wild-type (transparent representation) with (a) N501Y single mutated, (b) E484Q and L452R double mutated, and (c) N501Y, E484Q, and L452R triple mutated (solid representation) RBD structures. The mutated residues are shown in $v d W$ representation. The mutated RBD variants contain many secondary structure deviations from the wild type.

(N501Y, E484Q, and L452R) mutants generate large conformational switches that orient the RBD towards the N-terminal of ACE2 and the $\alpha$-helix region.

Hydrogen bonds are one of the most important intermolecular interactions and play key role in RBD and ACE2 binding. We have calculated the number of hydrogen bonds between RBD and ACE2 for wild type and mutated variants (Fig. 6). A hydrogen bond (H-bond) is defined to exist between a hydrogen atom involved in a polar bond and an electronegative acceptor (A) with a distance cut-off of $3.5 \AA$ and a D-H $\cdots$ A angle cut-off of $35^{\circ}$, where $\mathrm{D}$ is the donor. We have calculated the number of active $\mathrm{H}$-bonds during the last $100 \mathrm{~ns}$ of the $200 \mathrm{~ns}$ long trajectories. The average number of hydrogen bonds between RBD and ACE2 for wild type SARS-CoV-2 is very similar to that of other mutated variants. Our analysis shows that the number of $\mathrm{H}$-bonds between ACE2 and all the studied mutated variants of SARS-CoV-2 RBD is similar throughout the simulations. Moreover, the variant with the single N501Y mutation shows the least number of intact $\mathrm{H}$-bonds during the last $100 \mathrm{~ns}$ of the $200 \mathrm{~ns}$ long MD simulations. 

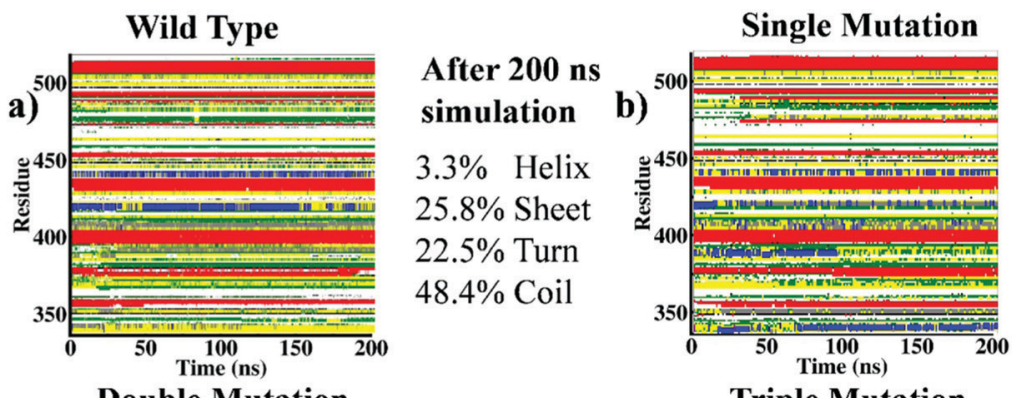

\section{After $200 \mathrm{~ns}$ simulation}

$2.7 \%$ Helix

$30.6 \%$ Sheet

$24.0 \%$ Turn

$40.4 \%$ Coil

$2.2 \% 3-10$ Helix

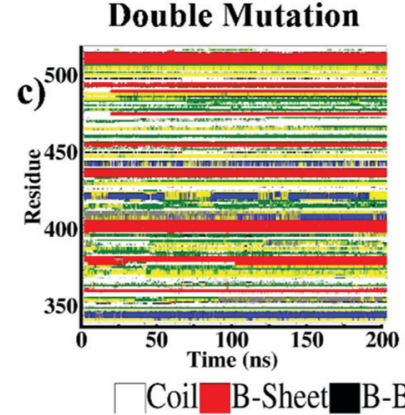

After $200 \mathrm{~ns}$
simulation

$24.7 \%$ Sheet

$22.5 \%$ Turn

$50.5 \%$ Coil

2.2\% 3-10 Helix

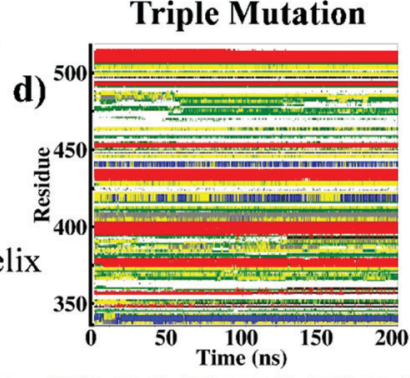

After 200 ns simulation

$3.3 \%$ Helix

$24.2 \%$ Sheet

$23.6 \%$ Turn

$48.9 \%$ Coil

Fig. 4 The secondary structure transition of (a) wild-type, (b) N501Y single mutated, (c) E484Q and L452R double mutated, and (d) N501Y, E484Q, and L452R triple mutated SARS-CoV-2 RBD after 200 ns simulations.

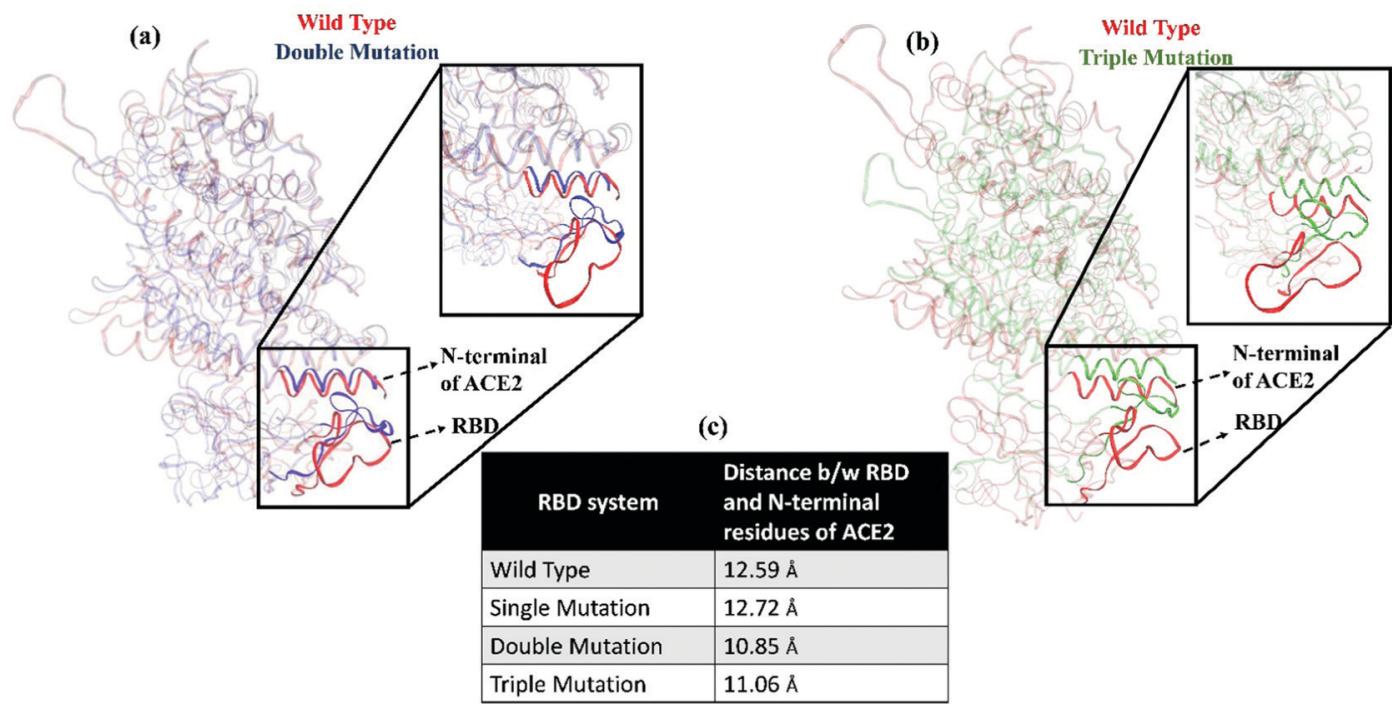

Fig. 5 Superimposed average structures of wild-type (red) with (a) E484Q and L452R double mutated (blue) SARS-CoV-2 and (b) N501Y, E484Q, and L452R triple mutant (green) where the N-terminal of ACE2 and the RBD residues in the vicinity are highlighted. (c) The average distance between the highlighted residues (first 15 residues of N-terminal of ACE2 and residues 469 to 489 for all the variant SARS-CoV-2 RBD studied in this work).

Using the MM-PBSA method, we compute the total binding energy of RBD with ACE2 (Fig. 7). The binding energy calculated using the MMPBSA method during the last $100 \mathrm{~ns}$ of the $200 \mathrm{~ns}$ long MD simulations shows a higher affinity for double (E484Q and L452R) mutant (-98.6 $\left.\pm 7.3 \mathrm{kcal} \mathrm{mol}^{-1}\right)$ than for wild type $\left(-59.7 \pm 9.6 \mathrm{kcal} \mathrm{mol}^{-1}\right)$, single (N501Y) mutant $\left(-58.0 \pm 8.0 \mathrm{kcal} \mathrm{mol}^{-1}\right)$ and triple (N501Y, E484Q, and L452R) mutant $\left(-79.8 \pm 9.2 \mathrm{kcal} \mathrm{mol}^{-1}\right)$. If this increased binding were to translate to increased infectivity, it may explain the high infection rate of the B.1.617 variant which recently caused the second wave of COVID infections in India.
To gain further insights into the mechanism causing these changes in the binding affinities, we have used the alanine scanning method (ASGB) to predict the hotspot residues in RBD involved in ACE2-RBD binding. The contributions from residues $417 \mathrm{~K}, 449 \mathrm{Y}, 486 \mathrm{~F}, 493 \mathrm{Q}, 500 \mathrm{~T}$ and $505 \mathrm{Y}$ are among the highest for the wild type ACE2-RBD. Large conformational changes are caused due to the mutations in RBD that increase the number of residues that interact with the ACE2 receptor. We find that $486 \mathrm{~F}$ is a key contributor to the binding energy of all the mutated variants of RBD studied. For the double (E484Q and $\mathrm{L} 452 \mathrm{R}$ ) mutated variant, the $498 \mathrm{Q}, 505 \mathrm{Y}$ and $486 \mathrm{~F}$ residues 


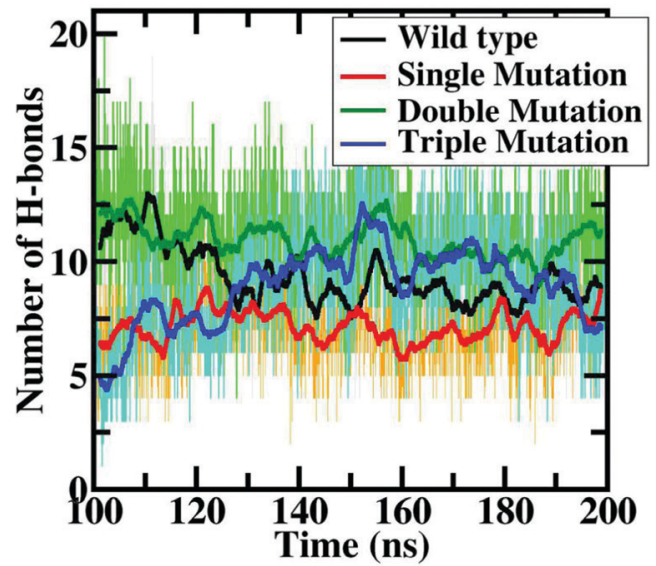

Fig. 6 A comparison of the number of $\mathrm{H}$-bonds between the ACE2 and RBD of wild-type, N501Y single mutated, E484Q and L452R double mutated, and N501Y, E484Q, and L452R triple mutated SARS-CoV-2 during the last $100 \mathrm{~ns}$ of $200 \mathrm{~ns}$ long MD simulations.

contribute the most, while for the triple (N501Y, E484Q, and L452R) mutated variant, the residues $486 \mathrm{~F}, 501 \mathrm{~N}$ and $505 \mathrm{Y}$ contribute the most. The total contribution from the hotspot residues is $-20.84 \mathrm{kcal} \mathrm{mol}^{-1}$ and single and double mutant were identified as $-29.94 \mathrm{kcal} \mathrm{mol}^{-1}$ and $-36.1 \mathrm{kcal} \mathrm{mol}^{-1}$. The triple (N501Y, E484Q, and L452R) mutated variant shows a higher binding affinity than wild type $\left(-33.06 \mathrm{kcal} \mathrm{mol}^{-1}\right)$. Thus, the double mutated (E484Q and L452R) variant shows the highest binding affinity. As shown in Table 2, the contribution of most of the residues of the mutated variants to the total binding energy is found to be higher than the contribution of the corresponding residues of wild type RBD. We note that ASGB does not consider the repulsive interactions which may lead to a difference between the computed binding energies using MMPBSA and ASGB. Nevertheless, the qualitative results obtained using ASGB agree well with MMPBSA and indicate that the double mutant (E484Q and L452R) RBD spike has
Table 2 Calculated binding free energy from key residues of RBD domain binding to ACE2. Energy values are in $\mathrm{kcal} \mathrm{mol}^{-1}$ and energy values above $-1.5 \mathrm{kcal} \mathrm{mol}^{-1}$ are considered

\begin{tabular}{|c|c|c|c|c|}
\hline \multirow[b]{2}{*}{ Residue } & \multicolumn{4}{|c|}{ Energy $\left(\mathrm{kcal} \mathrm{mol}^{-1}\right)$} \\
\hline & $\begin{array}{l}\text { Wild } \\
\text { type }\end{array}$ & $\begin{array}{l}\text { Single } \\
\text { mutation } \\
\text { (N501Y) }\end{array}$ & $\begin{array}{l}\text { Double mutation } \\
\text { (E484Q, L452R) }\end{array}$ & $\begin{array}{l}\text { Triple mutation } \\
\text { (N501Y, E484Q, } \\
\text { L452R) }\end{array}$ \\
\hline $408 \mathrm{R}$ & -1.53 & & & -1.55 \\
\hline $417 \mathrm{~K}$ & -2.98 & & & -1.57 \\
\hline $449 \mathrm{Y}$ & -3.27 & -2.77 & -3.71 & -2.77 \\
\hline $452 \mathrm{~L}$ & & -1.53 & & -1.53 \\
\hline $455 \mathrm{~L}$ & & -2.14 & -2.37 & -2.14 \\
\hline $456 \mathrm{~F}$ & & -1.61 & -1.74 & -1.61 \\
\hline $476 \mathrm{G}$ & & -1.67 & -1.73 & -1.67 \\
\hline $486 \mathrm{~F}$ & -2.42 & -4.07 & -4.48 & -4.07 \\
\hline $487 \mathrm{~N}$ & & -2.24 & -1.95 & -2.24 \\
\hline $489 Y$ & & -2.09 & -1.89 & -2.09 \\
\hline $493 Q$ & -2.67 & & & \\
\hline $495 Y$ & & & -1.78 & \\
\hline 498Q & -1.53 & & -5.24 & \\
\hline $500 \mathrm{~T}$ & -2.51 & -2.51 & -1.70 & -2.51 \\
\hline $501 \mathrm{~N}$ & & -3.82 & -2.86 & -3.82 \\
\hline $502 \mathrm{G}$ & & -1.67 & -1.88 & -1.67 \\
\hline $505 \mathrm{Y}$ & -3.93 & -3.82 & -4.77 & -3.82 \\
\hline Total & -20.84 & -29.94 & -36.1 & -33.06 \\
\hline
\end{tabular}

the highest binding affinity for ACE2 among the variants considered.

Next, we have compared our estimated binding energy data with the available experimental values. The experimental binding affinity values of mutated SARS-CoV-2 RBD with ACE2 are quite sparse. There are two major experiments-one by Laffeber et al. ${ }^{27}$ and the other by Kim et $a l^{28}$ - that reported the equilibrium dissociation constant $\left(K_{\mathrm{D}}\right)$ for several SARS-CoV-2 mutants. Binding energy is then calculated from $K_{\mathrm{D}}$ using the relation, $\Delta G=-R T \ln K_{\mathrm{D}}$. As shown in Table 3, the difference in binding energy $(\Delta \Delta G)$ of all the SARS-CoV-2 mutated variants is higher than that of the wild-type. This trend of higher binding energy in SARS-CoV-2 mutated variants than wild type is also seen in experimental studies (Table 3), although the simulation results

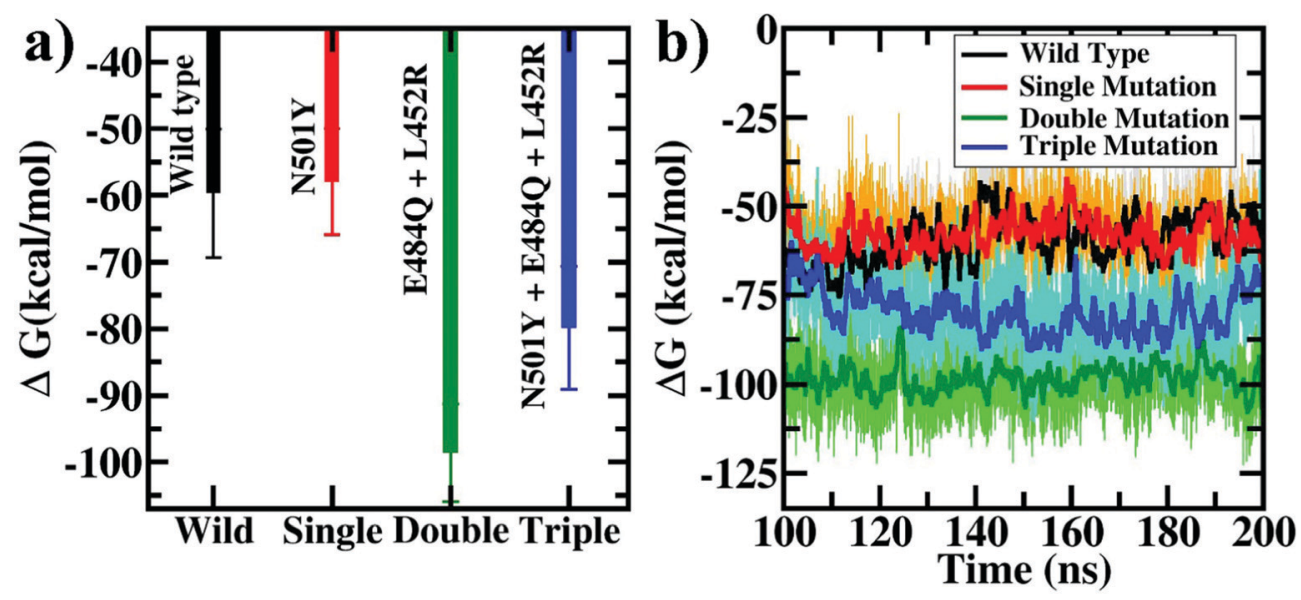

Fig. 7 (a) The binding energies of ACE2 with wild-type compared to that with N501Y single mutated, E484Q and L452R double mutated, and N501Y, E484Q, and L452R triple mutated RBD computed using MMPBSA simulations. (b) The variation of binding energy during the last 100 ns of the corresponding 200 ns long simulations. 
Table 3 Calculated differences in binding free $(\Delta \Delta G)$ energies of mutated SARS-CoV-2 variants and wild-type RBD and comparison with experiments. $\Delta \Delta G$ of different SARS-CoV-2 mutated variants is calculated with respect to the wild type

\begin{tabular}{lccr}
\hline System & $\Delta \Delta G$ from ASGB & $\Delta \Delta G$ from MMGB/PBSA & $\Delta \Delta G$ from experiment \\
\hline Single mutation (N501Y) & 9.1 & -1.7 & 1.16 (ref. 27), 0.50 (ref. 28) \\
Double mutation (E484Q, L452R) & 15.26 & 38.94 & 0.03 (ref. 27) \\
Triple mutation (N501Y, E484Q, L452R) & 12.22 & 20.19 & - N.A.-
\end{tabular}

overestimate the experimental data. Such overestimation of the binding energy is quite usual in simulations and is often related to the different force-fields used and the overestimation of the van der Waals interaction energy. ${ }^{26}$

\section{Conclusion}

We have performed extensive MD simulations of the binding of wild-type SARS-CoV-2 RBD, and its single (N501Y), double (E484Q and L452R) and triple (N501Y, E484Q, and L452R) mutated variants with human ACE2. We have used advanced free energy methods to calculate the associated binding energies. The double mutated (E484Q and L452R) variant shows the highest affinity followed by the triple mutant (N501Y, E484Q, and L452R), the wild-type, and the single (N501Y) mutant. A higher binding affinity arises due to the structural changes induced by mutations. Using the alanine scanning method, the residues of the RBD contributing the most to the binding energy difference were identified. The residue $486 \mathrm{~F}$ was identified as the key contributor in all the mutated variants we studied. For the double (E484Q and L452R) mutated variant, the 498Q, 505Y and 486F residues contribute the most, while for the triple (N501Y, E484Q, and L452R) mutated variant, the residues $486 \mathrm{~F}, 501 \mathrm{~N}$ and $505 \mathrm{Y}$ contribute the most. This study provides new mechanistic insights into the interactions between the wild-type and various mutated variants of SARSCoV-2 with the ACE2 receptor, enhancing our understanding of how the variants may acquire increased transmissibility. The structural insights gained may also help in designing sitespecific therapeutic molecules that may work against multiple variants.

\section{Conflicts of interest}

There are no conflicts of interest to declare.

\section{Acknowledgements}

We thank DST, India, for computational support through TUECMS computational facility. A. A. thanks MHRD, India, and Indian Institute of Science (IISc) for the generous fellowship. S. N thanks CSIR, India, and IISc for the generous fellowship. N. M. thanks IISc for support through the IoE (Institute of Eminence) postdoctoral scheme.

\section{References}

1 D. Paraskevis, E. G. Kostaki, G. Magiorkinis, G. Panayiotakopoulos, G. Sourvinos and S. Tsiodras, FullGenome Evolutionary Analysis of the Novel Corona Virus (2019-NCoV) Rejects the Hypothesis of Emergence as a Result of a Recent Recombination Event, Infect., Genet. Evol., 2020, 79, 104212, DOI: 10.1016/J.MEEGID.2020.104212.

2 W. Tai, L. He, X. Zhang, J. Pu, D. Voronin, S. Jiang, Y. Zhou and L. Du, Characterization of the Receptor-Binding Domain (RBD) of 2019 Novel Coronavirus: Implication for Development of RBD Protein as a Viral Attachment Inhibitor and Vaccine, Cell. Mol. Immunol., 2020, 17(6), 613-620, DOI: 10.1038/s41423-020-0400-4.

3 C. Huang, Y. Wang, X. Li, L. Ren, J. Zhao, Y. Hu, L. Zhang, G. Fan, J. Xu, X. Gu, Z. Cheng, T. Yu, J. Xia, Y. Wei, W. Wu, X. Xie, W. Yin, H. Li, M. Liu, Y. Xiao, H. Gao, L. Guo, J. Xie, G. Wang, R. Jiang, Z. Gao, Q. Jin, J. Wang and B. Cao, Clinical Features of Patients Infected with 2019 Novel Coronavirus in Wuhan, China, Lancet, 2020, 395(10223), 497-506, DOI: 10.1016/S0140-6736(20)30183-5.

4 N. Zhu, D. Zhang, W. Wang, X. Li, B. Yang, J. Song, X. Zhao, B. Huang, W. Shi, R. Lu, P. Niu, F. Zhan, X. Ma, D. Wang, W. Xu, G. Wu, G. F. Gao and W. Tan, A Novel Coronavirus from Patients with Pneumonia in China, 2019, N. Engl. J. Med., 2020, 382(8), 727-733, DOI: 10.1056/ NEJMOA2001017.

5 N. Maroli, B. Bhasuran, J. Natarajan and P. Kolandaivel, The Potential Role of Procyanidin as a Therapeutic Agent against SARS-CoV-2: A Text Mining, Molecular Docking and Molecular Dynamics Simulation Approach, J. Biomol. Struct. Dyn., 2020, 1-16, DOI: 10.1080/07391102.2020.1823887.

6 M. K. Gupta, S. Vemula, R. Donde, G. Gouda, L. Behera and R. Vadde, In Silico Approaches to Detect Inhibitors of the Human Severe Acute Respiratory Syndrome Coronavirus Envelope Protein Ion Channel, J. Biomol. Struct. Dyn., 2021, 39(7), 2617-2627, DOI: 10.1080/07391102.2020. 1751300.

7 J. Lan, J. Ge, J. Yu, S. Shan, H. Zhou, S. Fan, Q. Zhang, X. Shi, Q. Wang, L. Zhang and X. Wang, Structure of the SARS-CoV2 Spike Receptor-Binding Domain Bound to the ACE2 Receptor, Nature, 2020, 581(7807), 215-220, DOI: 10.1038/ s41586-020-2180-5.

8 R. Lu, X. Zhao, J. Li, P. Niu, B. Yang, H. Wu, W. Wang, H. Song, B. Huang, N. Zhu, Y. Bi, X. Ma, F. Zhan, L. Wang, T. Hu, H. Zhou, Z. Hu, W. Zhou, L. Zhao, J. Chen, Y. Meng, J. Wang, Y. Lin, J. Yuan, Z. Xie, J. Ma, W. J. Liu, D. Wang, W. Xu, E. C. Holmes, G. F. Gao, G. Wu, W. Chen, W. Shi and 
W. Tan, Genomic Characterisation and Epidemiology of 2019 Novel Coronavirus: Implications for Virus Origins and Receptor Binding, Lancet, 2020, 395(10224), 565-574, DOI: 10.1016/S0140-6736(20)30251-8.

9 K. Leung, M. H. Shum, G. M. Leung, T. T. Lam and J. T. Wu, Early Empirical Assessment of the N501Y Mutant Strains of SARS-CoV-2 in the United Kingdom, medRxiv, 2020, 26(1), 2002106, DOI: 10.1101/2020.12.20.20248581.

10 B. Luan, H. Wang and T. Huynh, Molecular Mechanism of the N501Y Mutation for Enhanced Binding between SARSCoV-R s Spike Protein and Human ACE2 Receptor, bioRxiv, 2021, 1454-1461.

11 S. Cherian, V. Potdar, S. Jadhav, P. Yadav, N. Gupta, M. Das, P. Rakshit, S. Singh, P. Abraham, S. Panda and N. Team, SARSCoV-2 Spike Mutations, L452R, T478K, E484Q and P681R, in the Second Wave of COVID-19 in Maharashtra, India, Microorganisms, 2021, 9, 1542, DOI: 10.3390/microorganisms9071542.

12 J. P. Sahoo, A. P. Mishra and K. C. Samal, Triple Mutant Bengal Strain (B.1.618) of Coronavirus and the Worst COVID Outbreak in India, Biot. Res. Today, 2021, 3(4), 261-265.

13 M. Hoffmann, H. Hofmann-Winkler, N. Krüger, A. Kempf, I. Nehlmeier, L. Graichen, A. Sidarovich, A.-S. Moldenhauer, M. S. Winkler, S. Schulz, H.-M. Jäck, M. V. Stankov, G. M. N. Behrens and S. Pöhlmann, SARS-CoV-2 Variant B.1.617 Is Resistant to Bamlanivimab and Evades Antibodies Induced by Infection and Vaccination, Cell Reports, 2021, 36(3), 109415, DOI: 10.1016/j.celrep.2021.109415.

14 Y. Liu, J. Liu, K. S. Plante, J. A. Plante, X. Xie, X. Zhang, Z. Ku, Z. An, D. Scharton, C. Schindewolf, V. D. Menachery, P.-Y. Shi and S. C. Weaver, The N501Y Spike Substitution Enhances SARS-CoV-2 Transmission, bioRxiv, 2021, DOI: 10.1101/2021.03.08.434499.

15 R. Yan, Y. Zhang, Y. Li, L. Xia, Y. Guo and Q. Zhou, Structural Basis for the Recognition of SARS-CoV-2 by FullLength Human ACE2, Science, 2020, 367(6485), 1444-1448, DOI: $10.1126 /$ SCIENCE.ABB2762.

16 D. A. Case, T. E. Cheatham, T. Darden, H. Gohlke, R. Luo, K. M. Merz, A. Onufriev, C. Simmerling, B. Wang and R. J. Woods, The Amber Biomolecular Simulation Programs, J. Comput. Chem., 2005, 26(16), 1668-1688, DOI: 10.1002/ JCC.20290.

17 W. L. Jorgensen, J. Chandrasekhar, J. D. Madura, R. W. Impey and M. L. Klein, Comparison of Simple Potential Functions for Simulating Liquid Water, J. Chem. Phys., 1998, 79(2), 926, DOI: $10.1063 / 1.445869$.
18 K. Lindorff-Larsen, S. Piana, K. Palmo, P. Maragakis, J. L. Klepeis, R. O. Dror and D. E. Shaw, Improved Side-Chain Torsion Potentials for the Amber Ff99SB Protein Force Field, Proteins: Struct., Funct., Bioinf., 2010, 78(8), 1950-1958, DOI: 10.1002/PROT.22711.

19 I. Suk Joung and T. E. Cheatham, Determination of Alkali and Halide Monovalent Ion Parameters for Use in Explicitly Solvated Biomolecular Simulations, J. Phys. Chem. B, 2008, 112(30), 9020-9041, DOI: 10.1021/jp8001614.

20 T. Darden, D. York and L. Pedersen, Particle Mesh Ewald: An $\mathrm{N} \cdot \log (\mathrm{N})$ Method for Ewald Sums in Large Systems, J. Chem. Phys., 1993, 98(12), 10089-10092, DOI: 10.1063/ 1.464397.

21 W. Humphrey, A. Dalke and K. Schulten, VMD: Visual Molecular Dynamics, J. Mol. Graphics, 1996, 14(1), 33-38, DOI: 10.1016/0263-7855(96)00018-5.

22 M. Ylilauri and O. T. Pentikäinen, MMGBSA as a Tool to Understand the Binding Affinities of Filamin-Peptide Interactions, J. Chem. Inf. Model., 2013, 53(10), 2626-2633, DOI: 10.1021/CI4002475.

23 S. Genheden and U. Ryde, The MM/PBSA and MM/GBSA Methods to Estimate Ligand-Binding Affinities, Expert Opin. Drug. Discov., 2015, 10(5), 449-461, DOI: 10.1517/17460441. 2015.1032936.

24 D. Huang, Y. Qi, J. Song and J. Z. H. Zhang, Calculation of Hot Spots for Protein-Protein Interaction in P53/PMIMDM2/MDMX Complexes, J. Comput. Chem., 2019, 40(9), 1045-1056, DOI: 10.1002/JCC.25592.

25 X. Liu, L. Peng, Y. Zhou, Y. Zhang and J. Z. H. Zhang, Computational Alanine Scanning with Interaction Entropy for Protein-Ligand Binding Free Energies, J. Chem. Theory Comput., 2018, 14(3), 1772-1780, DOI: 10.1021/ACS.JCTC. 7B01295.

26 Z. Li and J. Z. H. Zhang, Quantitative Analysis of ACE2 Binding to Coronavirus Spike Proteins: SARS-CoV-2 vs. SARS-CoV and RaTG13, Phys. Chem. Chem. Phys., 2021, 23(25), 13926-13933, DOI: 10.1039/D1CP01075A.

27 C. Laffeber, K. de Koning, R. Kanaar and J. H. G. Lebbink, Experimental Evidence for Enhanced Receptor Binding by Rapidly Spreading SARS-CoV-2 Variants, J. Mol. Biol., 2021, 433(15), 167058, DOI: 10.1016/J.JMB.2021.167058.

28 S. Kim, Y. Liu, Z. Lei, J. Dicker, Y. Cao, X. F. Zhang and W. Im, Differential Interactions Between Human ACE2 and Spike RBD of SARS-CoV-2 Variants of Concern, bioRxiv, 2021, DOI: 10.1101/2021.07.23.453598. 